\title{
Project-based Production and Project Management: FINDINGS AND TRENDS IN RESEARCH ON TEMPORARY SYSTEMS IN Multiple Contexts
}

\author{
Tinus Pretorius \\ Graduate School of Technology Management, University of Pretoria \\ Leon Oerlemans \\ Tilburg University, the Netherlands \\ Graduate School of Technology Management, University of Pretoria
}

\section{1}

Introduction

Globalisation is challenging almost every aspect of the political, economic, social and technological environment. Organisations, whether public or private, have to adapt their strategies and operations to stay competitive and efficient. Historically, organisations adopted project-based operations as a mode to stay competitive, although the applications tended to be the oneoff type of operations such as construction and system development projects (Edum-Fotwe \& McCaffer, 2000). As the world changed from an industrially driven to a more knowledge driven economy and the pace of continuous change became more intense, organisations adopted a project-based mode of operations on a broader scale. The knowledge economy lead to the creation of many service orientated industries. Organisations started facing portfolios of projects where the nature of these projects differed in technological complexity, urgency, customer value and social impact (Gutjahr \& Froeschl, 2013). Based on their experience with more technically orientated projects, organisations focused their attention more intensely on new project management methods, tools and processes and not necessarily on the human and organisational interfaces. This paradigm changed however, especially since the 1980s and more and more organisations adopted temporary organisational forms (Bakker, 2010) in order to improve their competitiveness. The contributions in this special edition of the South African Journal of Economic and Management Sciences have a common focus on the importance of the human and organisational interface of project-based operations on project success. The purpose of this concluding article is to analyse the findings and recommendations in these papers and to detect trends and future research opportunities in the field of project-based operations.

2

\section{Analysis of contributions}

\subsection{Macro perspectives}

Packendorff and Lindgren took a macro perspective on the processes of projectification. Based on previous literature, they refer to projectification as 'the development towards the use of projects for handling complex tasks and creative renewal in contemporary organisations', but at the same time describe this 'definition' as 'a narrow view of projectification' where research is mainly focussed on the contents and consequences of organisational re-structuring initiatives. Based on a thorough review of the relevant literature, Packendorff and Lindgren come to the conclusion that the basic weakness of the narrow view on projectification is that researchers exclude a view on projectification as a development characterised by for example bounded rationality, power and politics, cultural norms and constructs. They therefore argue that the research area should be extended from its current concern with the increased primacy of projects in contemporary organisational structures, to a concern for cultural and discursive processes in society. An important implication for research is that a new 
set of basic research assumptions is necessary, which implies that not only project researchers, but also scholars interested in for example organisational theory, should contribute to and complement future research on projectification. Such a multi-disciplinary approach will certainly generate new research questions related to both the individual and the organisation in the project-based operations environment.

\subsection{Meso perspectives}

In the contribution on project governance, Bekker argues that current research literature on project governance lacks a broader, multifirm and multi-project perspective with a common understanding of the definitions, frameworks and contexts. The single-firm governance perspective concerns itself with intra-organisational projects and therefore tends to practice governance principles solely at a technical level. The multi-firm project governance approach however, is more concerned with the contractual level of cooperation, while the large capital project governance perspective with its temporary organisations approach, tends to establish governance principles at the institutional level. Given these different governance perspectives, it is therefore necessary that researchers focus on the alignment of project governance principles among the three organisational categories. The research challenge here is to broaden the perspectives from the technical level of control in the single-firm school, to align with the contractual level of control in the multi-firm school. With the large capital project school, control becomes a complex activity within a cosmopolitan composition of various cultures and values, organisational structures and many more. In line with the recommendation of Packendorff and Lindgren, Bekker also views the involvement of researchers from multidisciplinary research backgrounds in the project governance research area as important condition for future research, especially concerning the large capital project school within a highly complex and multi-dimensional organisational environment. A research approach as investigated from the broad projectification research view proposed by Packendorff and Lindgren, where project governance principles are aligned between the different schools of thought, could also bring new perspectives on how future temporary organisations should deal with project governance.

Jerbrant's paper focuses on the changes and maturation in the management and organisation of project-based organisations within multiproject environments. The aim of her article was to deepen our understanding of how the management of project-based organisations could evolve in a flow between structuring administration and managing potential uncertainty. The point of departure is how standardised management processes evolved into the more strategic perspectives on the entire project portfolio and the ultimate link to the overall business goal. Uncertainty management is emphasised with its resulting complexities and impact on restructuring activities. Therefore, a maturation model that visualises the projectbased organisation, oscillating between restructuring and uncertainty management is proposed. The outcome of this research is of importance since it takes a broader perspective than only the project management view and ultimately links the restructuring activities to the business goals of the organisation. Jerbrant thus proposes future research to focus at least on (i) coordination in the projectified part of the business where integration of several functional areas is needed, and (ii) coordination to align the project portfolio with the overall business strategy. Studies on further development of the maturation model as well as on uncertainty management in different types of project-based organisations should also be on the future research agenda.

\subsection{Micro perspectives}

Van Kessel et al. focused their research on a specific case in the micro environment, namely an academic institution where creative research paper output is an important strategic goal of the organisation. Their research examined the relationship between perceptions of organisation culture, social embeddedness of the papers' authors and the paper project outputs. The finding that social ties with colleagues both inside and outside their departments, but in the same academic institution, matter for outcomes is a causal step linking organisational values and norms to creative outputs, and is of importance to the broader project-based production community. Linking these research 
findings to those of (i) Chan, and (ii) Chang and Yeh, brings interesting new perspectives that could help us to develop deeper insights into the needs of project-based operations.

Chan studied the impact of multiple project team membership on innovative project performance and found that an inverted u-shaped relationship exists between multiple team membership and individual creative performance. This finding is explained by the more diverse sources of ideas that the individual encounters, although too many diverse ideas have a negative effect on the individual's creative performance. This finding of Chan corresponds with the conclusion of Van Kessel et al. that 'no project is an island'. Chan also found that initially multiple team membership has a positive linear relationship with team performance (and at higher levels a negative effect). These finding again correspond with the conclusion of Van Kessel et al. that 'no project is an island', assuming that a project is seen as a team consisting of members. Overall, both papers emphasize that the project environment matters for performance.

Chang and Yeh contributed to the research on project-based production by examining the relationships between intra project team disagreement, conflict communication and team performance in cross-functional new product project teams and its consequences on decisionmaking comprehensiveness. The findings of this research are relevant in that it gives further insight into the nature of specific social process characteristics between project team members and their effects. First, they found a concave relationship between intra-team task disagreement and decision-making comprehensiveness and explain their finding by suggesting that too little conflict within project teams discourages the combination of different points of view and diverse information sources to create a new knowledge base in the decision-making process. Too much intra-team task disagreement also decreases the decisionmaking comprehensiveness. Second, they found that decision-making comprehensiveness does not appear to be the most fruitful source of creative ideas for innovation. Third, they found that collaborative communication has a significant and negative effect on innovativeness and suggest further research to examine this specific finding. From the findings of Chang and Yeh it can be concluded that social processes between individual project team members and between project teams are indeed important but that the social ties should take the project goal into consideration, otherwise the outcome will have a negative effect on the project goals.

\section{3}

\section{Future research}

Packendorff and Lindgren propose that future research on projectification should actively employ a view of projects and project-based organising as cultural and discursive phenomena. They encourage organisation theory scholars to find studies of projectification most useful in developing new theoretical notions on, for example post-burocratic organisations, virtual organisations and entrepreneurial processes. On the meso level, Bekker proposes further research on the development of project governance frameworks for projects spanning across country borders and incorporating different value systems, legal systems, corporate governance guidelines, religions and business practices. Also on the meso level Jerbrant proposes several topics for future research, namely (i) coordination to integrate several different functional areas in the part of a business that is projectified, (ii) coordination to align the project portfolio with the overall business strategy, (iii) the strategic link between business development and both level of structuring and uncertainty management, (iv) further development of the maturation model she proposed and (v) the content of uncertainty management in different types of project-based organizations. On the micro level Van Kessel et al. propose three topics for future research, namely (i) examine whether organizational culture affects the number of social ties, or vice versa, (ii) the extent to which the strength of ties mediates the relationship between organizational culture and creative output and (iii) the mediating role of social embeddedness in the relationship between organizational culture and creative output. Based on her work on multiple project team membership and performance Chan proposes four topics for future research. 
Firstly, other propositions proposed by O'Leary et al. (2011), for example 'variety' (i.e. diversity in tasks, technologies, locations, and so forth) can be empirically tested so that the impact of multiple team membership can be fully examined. Secondly, in order to mitigate the self-assessed individual performance bias problem, multisource measurement procedures can be used. Thirdly, other types of projects than only engineering-related project teams with different tasks can be investigated to determine the impact of multiple team membership on performance. Finally, the impact of multiple team membership on performance at organizational level can be explored because pressures on teams, team members and their leaders might be more severe. For future research, Chang and Yeh propose that a longitudinal research design is used to better validate the causal sequences that are proposed in their study. They also recommend that for future research, criterion variables are assessed using a comprehensive measure that contains several subjective measures such as innovativeness and constraint adherence, as well as objective financial performance measures.

A common thread in the proposed topics for future research is that traditional project management approaches and tools should be complemented by insights from the social sciences, in other words, multi-disciplinary research in which an engineering perspective is combined with a social science perspective.

\section{References}

BAKKER, R.M. 2010. Taking stock of temporary organizational forms: a systematic review and research agenda. International Journal of Management Reviews, 12(4):466-486.

EDUM-FOTWE, F.T. \& MCCAFFER, R. 2000. Developing project management competency: perspectives from the construction industry. International Journal of Project Management, 18(2):111-124.

GUTJAHR, W.J. \& FROESCHL, K.A. 2013. Project portfolio selection under uncertainty with outsourcing opportunities. Flexible Services and Manufacturing Journal, 25(1-2):255-281.

O'LEARY, M.B., MORTENSEN, M. \& WOOLEY, A.W. 2011. Multiple team membership: A theoretical model of its effects on productivity and learning for individuals and teams. Academy of Management Review, 36(3):461-478. 